\title{
Binaural masking of tones by tones: Effects of uncertainty regarding the phase angle of addition of signal to masker
}

\author{
E. OSMAN, H. TZUO, and P. L. TZUO \\ Brooklyn College, City University of New York, Brooklyn, New York
}

\begin{abstract}
This paper compares performance in tone-on-tone coherent binaural masking for conditions with and without listener knowledge concerning the phase angle of addition of the signal to the masker. Uncertainty was introduced by randomizing the four phase angles: $0,45,90$, and $135 \mathrm{deg}$. Stimuli were 125 -msec tone bursts at $250 \mathrm{~Hz}$. The results showed a clear decrement in detection performance under the uncertainty condition at $135 \mathrm{deg}$, with weaker evidence for decrements at other phase angle values. This supports the assumption that the decision process was a function of the phase angle in previous studies of binaural tone-on-tone masking.
\end{abstract}

One major class of published research on binaural detection concerns coherent binaural masking, in which binaural signals and maskers originate from the same source and have identical spectra during the appropriate presentation interval. Tonal, narrowband noise and broad-band noise waveforms have been studied. Masker waveforms were interaurally identical (condition MO), while the signal was presented with an interaural phase shift of either $0 \mathrm{deg}$ (condition SO) or $180 \mathrm{deg}$ (condition $\mathrm{S} \pi$ ), independently of frequency. The signal is added to the masker after a phase shift, which, for convenience, is designated $\alpha$ (alpha) at one ear (and, consequently, $180 \mathrm{deg}-\alpha$ at the other ear for the $\mathrm{S} \pi$ condition). The results are reported in some form representing the signal-to-masker ratios required for a constant level of detectability as a function of $\alpha$. The relevant papers include Grantham and Robinson (1977), Hafter and Carrier (1970), Jeffress and McFadden (1971), McFadden, Jeffress, and Ermey (1971), McFadden, Jeffress, and Lakey (1972), Osman, Tzuo, and Tzuo (1980), Robinson, Langford, and Yost (1974), Wightman (1971), and Yost (1972). We are interested in evaluating how well various binaural processing models can be used to describe quantitatively the results of such coherent masking experiments. However, those results are heavily dependent on practice effects and show large individual differences, in which the variability for MO-S $\pi$ of the dependency of threshold on $\alpha$ is great across subjects, as well as across signal frequencies for a given listener. Below

\footnotetext{
This research was supported by grants from the United States Department of Health, Education, and Welfare, Public Health Service (Grant R01 NS10843) and the PSC-BHE Research Award Program of the City University of New York. We wish to thank Claire Spettell and Chris Zias for serving as listeners. Address correspondence to E. Osman, Department of Psychology, Brooklyn College, City University of New York, Brooklyn, New York 11210.
}

$1,000 \mathrm{~Hz}$, the results are generally highly asymmetrical about $\alpha=90 \mathrm{deg}$, and even for $\alpha<90 \mathrm{deg}$ (where interaural time and intensive differences are consonant), the thresholds for different listeners may increase as well as decrease with increasing $\alpha$. Our concern in this paper is with the shape of the function relating detection performance (threshold) to $\alpha$ in the antiphasic (MOS $\pi$ ) condition for $0 \mathrm{deg} \leqslant \alpha \leqslant 135 \mathrm{deg}$. (Above $\alpha=90 \mathrm{deg}$, interaural time and intensive differences are dissonant.) The form of the results for the homophasic (MOSO) condition generally conforms to what would be expected if detection were based on the energy increment's resulting from the addition of the signal to the masker at either ear. In the MOS $\pi$ conditions, however, the addition of the signal to the masker creates an interaural intensive difference as well as an interaural phase shift, and detection performance is usually far too good to be due to the small change in monaural energy that results from the addition. Figure 1 displays a vector diagram for this antiphasic stimulus configuration along with definitions of relevant derived quantities. The Hafter and Carrier (1970) "lateralization" model for tone masked by tone presumes detection at threshold to require constancy of the magnitude of a weighted sum of resultant interaural amplitude and phase (time) differences. It does describe some of the empirical MOS $\pi$ results to a reasonable degree of satisfaction, but only for $0 \mathrm{deg} \leqslant \alpha \leqslant 90 \mathrm{deg}$, where it yields functions of $20 \log (\mathrm{S} / \mathrm{M})$ at threshold vs. $\alpha$ that are concave upward (and asymmetrical about $90 \mathrm{deg})$. The model for subjective lateral position proposed by Stern (1976) can be applied to binaural coherent masking, but the predictions are identical to those of the lateralization model. Those predictions yield poor fits to the data when interaural time and amplitude shifts are dissonant. Domnitz (1975) has also modeled some binaural coherent masking re- 


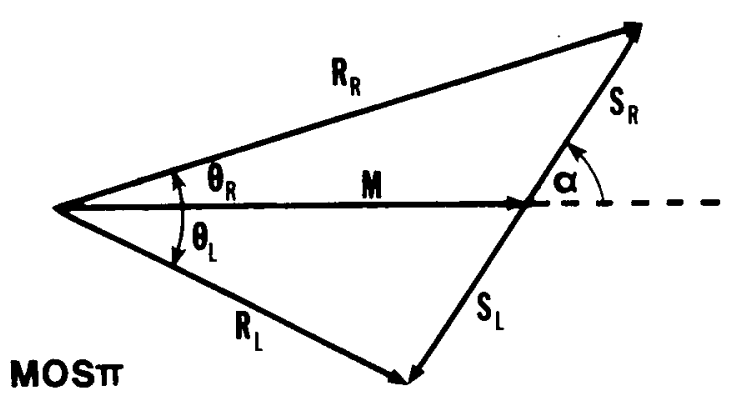

$I=M^{2}$

$\Delta I=S^{2}+2 m S \cos \alpha$

$\mathbf{l}+\Delta \mathbf{l}=\mathbf{R}^{2}$

$\Delta I / I(d B)=10 \log \left[\left(S^{2}+2 M S \cos \alpha\right) / M^{2}\right]$

$(I+\Delta I) / I(d B)=10 \log \left(R^{2} / M^{2}\right)$

$\Delta \theta=\theta_{R}+\theta_{L}$

$\Delta R=10 \log \left(R_{R}^{2} / R_{L}^{2}\right)$

Figure 1. Vector diagram showing the antiphasic MOS $\pi$ stimulus configuration and definitions of relevant derived quantities. $M$ is the amplitude of the masker at either ear, $S_{R}$ and $S_{L}$ are the signal amplitudes for right and left ears, $\alpha$ is the phase angle of addition of signal to masker (defined for the right ear). $\mathbf{R}_{R}$ and $\mathbf{R}_{\mathbf{L}}$ are the resultant vector sums at the respective ears, and $\theta_{\mathbf{R}}$ and $\theta_{L}$ are the respective phase shifts that result from the addition of the signal to the masker.

sults, using a subjective lateral position variable and a subjective loudness variable plus acoustic imbalance. The models of both Domnitz and Stern were produced to extend the binaural processing theory advanced by Colburn (1973, 1977). His "auditory nerve based" model, which uses a weighted sum of the outputs of $n$ coincidence counters with different internal delays, cannot by itself be applied to binaural coherent masking. Similarly, both the "equalization-cancellation" model and the "correlation" model, in their currently published forms (Durlach, 1972; Osman, 1971, 1973; Osman, Schacknow, \& Tzuo, 1973; Osman et al., 1975), cannot describe the binaural coherent masking results. However, these two models are special cases of a general "correlation-energy" model based on a weighted sum of monaural energy and binaural cross-correlation quantities, and as such can be readily modified using reasonable assumptions to provide descriptions of the binaural coherent masking results at least as satisfactory as those of the previously mentioned models. The assumptions could be in the form of interaural amplitude and/or interaural phase imbalances. Still another option is to use an assumption that was previously incorporated into both the equalizationcancellation and correlation models (Durlach, 1972; Osman, 1971). This was that the auditory system can transform the waveforms in the two auditory channels by inserting a compensating phase shift to bring the interaural phase difference to 0 deg early in the processing sequence, which yields the appropriate form for the dependency of detection threshold on $\alpha$. Nevertheless, to the extent that variations in listener decision strategy are reflected in the learning effects and individual differences evident in the dataor, in other words, that receiver operation is not "hard-wired" in the auditory system-any models such as those discussed above would be difficult to test, in spite of their flexibility. The experiment reported here directly addresses this problem.

The general rationale underlying our study of coherent binaural masking with (and without) $\alpha$ uncertainty is as follows. In the relevant $\alpha$ experiments that have been reported so far, listeners worked under constant interaural relations throughout each block of psychophysical trials, so that zero trial-totrial uncertainty regarding those interaural relationsor, specifically, the value of $\alpha$-could be assumed. (Listeners were also instructed to use different strategies for homophasic, as compared with antiphasic, conditions.) With extensive training preceding the testing of performance under conditions in which the listener has maximum prior information regarding the relevant stimulus parameters, at least in the sense that those parameters are fixed for experimental sessions of reasonable length, we should expect to find optimal performance. However, in considering the theoretical problem alluded to earlier, it is possible that even optimal listener strategy is a function of those stimulus parameters. Specifically for the case at hand, there may be no single operation on input waveforms that will generate best performance independently of specification of $\alpha$. Actually, the effects of uncertainty on performance under different conditions (values of $\alpha$ ) cannot be predicted without specification of an adequate statistical model. For any of the possible binaural models, if the different random variables to be summed have different variances, that will determine the performance decrements when the weightings shift under uncertainty conditions. The problem is readily conceptualized by thinking in terms of the mean to standard deviation ratio of a linear combination of random variables as a function of changing coefficients. Thus, for any model in which choice of weights for components of an overall decision variable may be considered one form of representation of decision strategy, no single set of weights might suffice to explain the data from any well-trained listener with full certainty regarding $\alpha$. Generally, experiments with input parameter uncertainty from trial to trial (or within trials) would be likely to force listener performance (decision strategy) to be independent of those input parameters. Then, if a particular model is applicable, a single choice of decision variable (i.e., weighting of its com- 
ponents) should suffice to describe the data. Furthermore, a comparison of model parameters under conditions of certainty and uncertainty should be useful for further development of such models. In view of the possibility of multiple decision variables, note that the performance under uncertainty conditions would be most interesting for those particular individuals showing best performance under conditions of certainty.

The recent paper of Osman et al. (1980) reports an experimental study of binaural coherent masking with varying levels of masker intensity in the conceptual framework of intensity discrimination. It includes a detailed review and discussion of the most relevant data from earlier $\alpha$ experiments. Grantham and Robinson (1977) reported binaural coherent masking results for static and dynamic (via amplitude modulation) interaural differences, again with the order of data collection for different values of $\alpha$ fixed (i.e., not counterbalanced, minimum uncertainty). Grantham and Robinson also questioned whether the large individual differences in performance reflect different "wiring" in different individuals or the modification of choice of decision variable through training.

This experiment involves a replication of and supplement to experimental work reported in the literature for low-frequency tone-on-tone binaural masking. In terms of our choice of stimulus parameters (frequency, duration, level, and gating mode), the replication is most directly comparable to Hafter and Carrier (1970) and Yost (1972). The supplement introduces listener uncertainty with regard to $\alpha$-or, more directly, the interaural phase and amplitude differences produced by $\alpha$.

\section{PROCEDURES}

For both fixed and random a conditions (described below), signals and maskers were synchronously gated $250-\mathrm{Hz}$ tones of 125 -msec total duration with $10-\mathrm{msec}$ rise/fall times. The masker level was fixed at $70 \mathrm{~dB}$ SPL. The tones were generated by a single oscillator and were then split into electrically isolated channels for signal and masker. Signal-masker phase shifts $(\alpha)$ were produced as time delays using a Reticon Analog Delay Line (SAD1024). The signal was gated by a Grason-Stadler electronic switch (829E) to overlap one or the other of the two observation intervals on each trial. Signal and masker were split again into two isolated channels for left and right earphones, and one of the resulting signals was inverted to produce the $\mathbf{S} \pi$ conditions, or maximally attenuated for a monaural condition. Signal and masker were then summed for each earphone using resistive mixers. Both sums were then gated by a two-channel Grason-Stadler electronic switch (829E) for the duration of each observation interval and were then passed to the two TDH-39 earphones via impedance-matching transformers. Attenuation and filtering were employed at various points throughout the network. Note that time shifts were accomplished prior to gating so that left- and right-ear stimuli were properly synchronized. All stimulus parameters were carefully and regularly calibrated by measurements made at the input to the earphones using a dual-beam oscilloscope, a Ballantine True RMS voltmeter $(320 \mathrm{~A})$, and a Krohn-Hite phase meter (6200A). Lis- tening was done in an IAC double-walled soundproof chamber. The psychophysical paradigm was $2 \mathrm{ATFC}$ (two-alternative temporal forced choice), in which each trial was initiated by the listener and consisted of a 1,200-msec waiting period, followed by two observation intervals (of $125 \mathrm{msec}$ each) separated by $500 \mathrm{msec}$, and then a response interval that was terminated by the listener's vote (buttonpress) indicating which interval was judged to contain the signal. The signal was present (added to the masker) in only one of the two observation intervals, with equal probability for each. Indicator lights were used to mark the observation intervals and to provide trial-by-trial feedback regarding correct or incorrect decisions. Each trial block consisted of 80 trials.

For the first stage of the experiment, which replicates previous work, complete psychometric functions were determined for each of four listeners (including two of the authors) for $\alpha=0,45,90$, and $135 \mathrm{deg}$. For this purpose, all stimulus parameters (including signal level and $\alpha$ value) were fixed for each block of trials. For each value of $\alpha$, signal levels were varied in 2-dB steps in descending order over different blocks to generate psychometric functions, and this was replicated four times, so that there were 320 trials for each point on each psychometric function for each listener. Data for different values of $\alpha$ were collected in a balanced order. For each listener, all the data from the four replications at each signal level and value of a were combined, and the proportion of trials for which the listener correctly decided that the signal was in the first interval and the proportion of trials for which the listener incorrectly decided that the signal was in the first interval were used as hit and false-alarm rates. Then the two corresponding standard normal scores were determined, and their sum divided by $\sqrt{2}$ was used as $d^{\prime}$. A linear psychometric function of the form $\log \mathrm{d}^{\prime}=\mathrm{c}+\mathrm{k} \log \mathrm{S}_{\mathrm{R}}{ }^{2}$ (Egan, Lindner, \& McFadden, 1969) was fitted by least squares to the data over signal levels $\left(S_{R}\right)$. Intercepts for $\mathrm{d}^{\prime}=1$ were used as thresholds (corresponding to a maximum proportion correct of .76). These procedures for the first stage were run formally only after a considerable number of practice sessions, and they were replicated in their entirety three times: in June 1979, August 1979, and July 1980. Psychometric functions were similarly generated to determine both monaural thresholds $\left(\mathrm{S}_{\mathrm{R}}\right.$ and $\mathrm{S}_{\mathrm{L}}$ ) and the binaural diotic threshold $(\mathrm{SO})$, with the diotic masker (MO), in June 1979.

For the entire second (test) stage of the experiment, which is the crucial supplement, signal levels were fixed at threshold values estimated from the last replication of the first stage (July 1980). The listeners' task was identical to that of the first stage. For the first "fixed $\alpha$ " condition, four blocks of 80 trials each were run consecutively for each value of $\alpha$. A $10-$ to 20-min rest period was the minimum requirement before switching values of $\alpha$. Data were collected for the four values of $\alpha$ in the order: $0,45,90$, $135 \mathrm{deg}$. Following completion of this, on the following day the "random $\alpha$ " condition was begun. Here the value of $\alpha$ (and its corresponding fixed signal level) was selected at random from the set of four $\alpha$ values on a trial-by-trial basis. There were no restrictions on the randomization other than that the four pairs of values of $\alpha, S_{R}$ be equally likely. A total of 16 blocks of 80 trials each were run, so that each data point would be based on approximately 320 trials. On the day following completion of the random a runs, another fixed $\alpha$ condition was run as before. In this case, the only procedural change was to collect the data for the four values of $\alpha$ in reverse order: $135,90,45$, and $0 \mathrm{deg}$. Data processing for this stage of the experiment amounted to determining values of $\mathrm{d}^{\prime}$ as specified above.

\section{RESULTS AND DISCUSSION}

We have displayed the results of the first stage of the experiment in Figure 2 in the form of $\Delta \mathrm{I}_{\mathrm{R}} / \mathrm{I}(\mathrm{dB})$ $=10 \log \left(\mathbf{S}_{\mathbf{R}^{2}}+2 \mathrm{~S}_{\mathrm{R}} \mathrm{M} \cos \alpha\right) / \mathrm{M}^{2}$ as a function of $\alpha .^{1}$ The most relevant properties of these results are that 


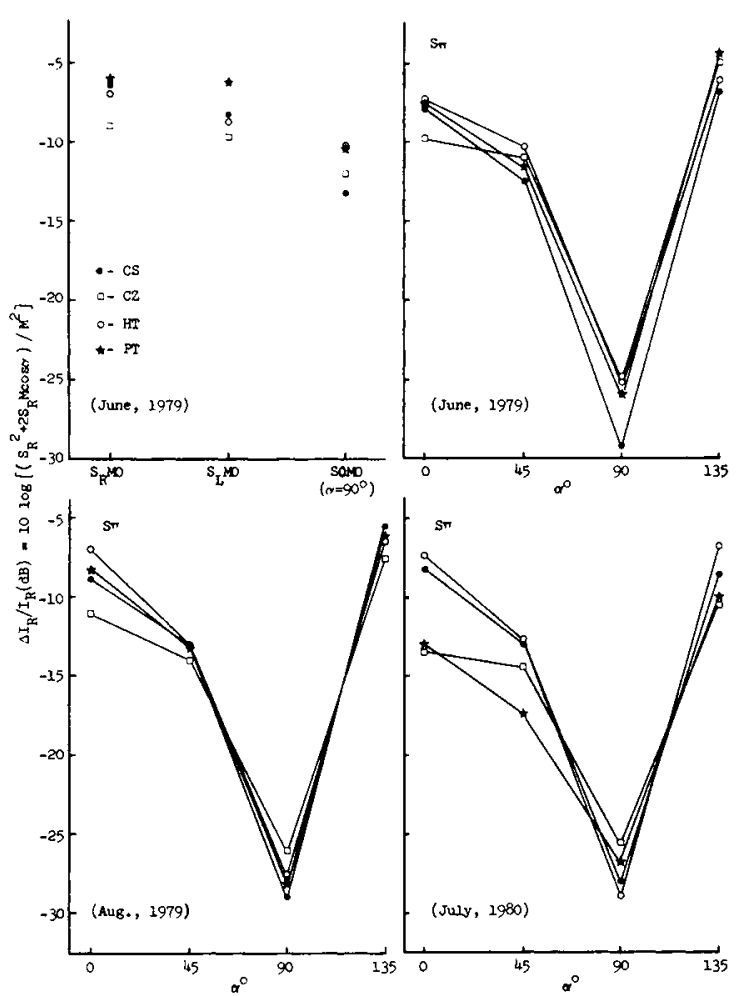

Figure 2. Threshold values of $\Delta I_{R} / I_{R}(d B)=10 \log \left[\left(S_{R}{ }^{2}+2 S_{R} M\right.\right.$ $\left.\cos \alpha) / M^{2}\right]$ vs. $\alpha$ for the binaural condition $M O S \pi$. Results are also shown for $S_{R} M O, S_{L} M O$, and SOMO with $\alpha=90$ deg. Data were collected during the months indicated.

they compare favorably with the values obtained from other studies (as summarized in Table 7 of Osman et al., 1980). Examination of the graphs shows some small improvements in performance over time that are scattered over listeners and values of $\alpha$. We now assume that the goal of optimizing performance has been met to at least a reasonable degree of satisfaction. ${ }^{2}$

Our main concern in this paper is with the second stage of the experiment. Those results are displayed in Figure 3, in which we have chosen to plot the maximum percentage of correct decisions in 2ATFC (which is a transform of $d^{\prime}$ ) on the ordinate. ${ }^{3}$ The three conditions on the abscissa are in order of their occurrence, where the fixed $\alpha$ (before) condition and the fixed $\alpha$ (after) condition are those that immediately preceded and succeeded the random $\alpha$ condition. Although the averages over listeners appear in the graphs, the patterns of individual listener data for the four values of $\alpha$ are clearly of greatest importance and should be examined carefully whenever the average results are not representative of all listeners.

The most impressive result shown in Figure 3 is that uncertainty regarding $\alpha$, as we have defined it, produces a rather large decrement in performance for the setting of $\alpha=135 \mathrm{deg}$ consistently for all four listeners. All of the functions at $135 \mathrm{deg}$ are V-shaped. Three such V-shaped functions appear at $\alpha=45 \mathrm{deg}$, although the magnitude of the effect is smaller. One listener shows a V function for $\alpha=0 \mathrm{deg}$, where the signal level was apparently set higher than planned. These last four functions suggest that uncertainty has significantly interfered with performance. There is one other small V-shaped function at $\alpha=90 \mathrm{deg}$, but the drop for random $\alpha$ is not impressive. There are no cases in which performance under the random condition appears to be significantly better than performance under both fixed conditions, the most likely such case occurring at $\alpha=0 \mathrm{deg}$. Furthermore, we must consider that several functions show performance under the two fixed $\alpha$ conditions to differ by fairly large magnitudes. [Our inspection of these graphs is guided by the consistencies that we perceive in them. The effects of uncertainty at $\alpha=135 \mathrm{deg}$ are decrements in performance of the order of .15 or more for $\mathrm{P}_{\max }(\mathrm{C})_{2}$. For other values of $\alpha$, the large changes are of the order of .10.] There is no doubt that uncertainty regarding $\alpha$ produces a large decrement in performance at $\alpha=135 \mathrm{deg}$, and this is com-

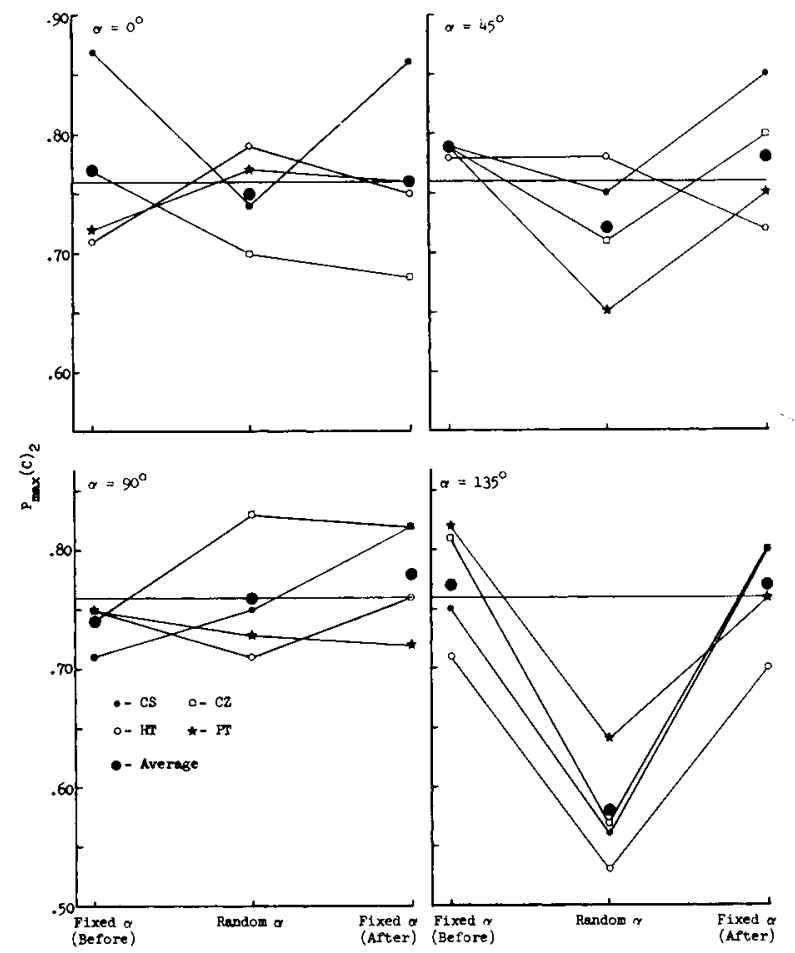

Figure 3. Values of percent correct in $2 \mathrm{ATFC}$ corrected to maximum for conditions with $\alpha$ fixed before randomization, $a$ randomized over the set of four values, and $\alpha$ fixed after randomization. Each graph is for the value of $\alpha$ indicated, with the data for random $\alpha$ derived from only the appropriate individual trials. The data were collected in July 1980 , following the collection of the data for the last panel of Figure 2. (The large circles are averages over the four listeners, included for the reader's convenience.) 
patible with the arguments offered in our introduction. For $\alpha=45 \mathrm{deg}$, there may be significant effects of uncertainty in the same sense, when there is a drop in performance for the random as compared with the fixed conditions. Furthermore, it is conceivable that the introduction of the random condition is sufficiently confusable for the listener to cause a change in detection strategy that persists, so that there is a sizable difference in performance between the two fixed conditions (before and after). To seriously analyze the results along these lines would require both a specification of decision strategies available to the listener in quantitative form and a reasonably low level of variance in the data. We are not yet ready to achieve this. However, it is important to remember that our main variable, $\alpha$, cannot be sensed directly by the auditory system. The phase angle of addition of signal-to-masker, $\alpha$, produces ongoing interaural phase differences and interaural amplitude differences that are often presumed to be the basis of the binaural detection process. For $\alpha=0 \mathrm{deg}$, there is only an interaural amplitude difference, while for $\alpha=90 \mathrm{deg}$, there is only an interaural phase difference. Both exist for $\alpha=45$ or $135 \mathrm{deg}$, where the resultant of larger amplitude leads in phase at $\alpha=$ $45 \mathrm{deg}$ (consonance) and the larger resultant lags in phase for $\alpha=135 \mathrm{deg}$ (dissonance). In relation to the general functioning of the auditory system for sound localization, the dissonance condition is a most unnatural task, and therefore may be most subject to the effects of uncertainty. Evidence in support of the large individual differences in the data for nondissonance conditions of Figure $3(\alpha \leqslant 90 \mathrm{deg})$ exists throughout the literature in that subjects have been categorized in terms of the tendency to be differentially sensitive to interaural phase and interaural amplitude (Grantham \& Robinson, 1977; Hafter \& Carrier, 1972; Jeffress \& McFadden, 1971; McFadden et al., 1971; McFadden et al., 1972; Osman et al., 1980).

Thus, we shall limit our summary interpretation of this study to the following. Listener strategy in binaural coherent masking experiments with lowfrequency tones is dependent on the phase angle of addition of signal to masker, when there is information allowing such a dependency. Specifically, optimal detection strategies are different for conditions in which interaural amplitude and phase differences are consonant as compared with conditions in which they are dissonant. There remains the possibility that optimal detection strategy varies with changes in interaural parameters within the range of consonance to a significant degree. Consequently, it may be inappropriate to reject any model of binaural processing because it cannot parsimoniously describe the contour relating performance level to $\alpha$.

\section{REFERENCES}

Colburn, H. S. Theory of binaural interaction based on auditorynerve data. I. General strategy and preliminary results on interaural discrimination. Journal of the Acoustical Society of America, 1973, 54, 1458-1470.

Colburn, H. S., \& Durlach, N. I. Model of binaural interaction. In E. C. Carterette \& M. P. Friedman (Eds.), Handbook of perception (Vol. 4): Hearing. New York: Academic Press, 1977.

Domnitz, R. H. Lateral position and loudness in binaural experiments. Unpublished doctoral dissertation, M.I.T., 1975.

Durlach, N. I. Binaural signal detection: Equalization and cancellation theory. In J. V. Tobias (Ed.), Foundation of modern auditory theory (Vol. 2). New York: Academic Press, 1972.

Egan, J. P., Linder, W. A., \& McFadden, D. Masking-level differences and the form of the psychometric function. Perception \& Psychophysics, 1969, 6, 209-215.

Grantham, D. W., \& Robinson, D. E. Role of dynamic cues in monaural and binaural signal detection. Journal of the Acoustical Society of America, 1977, 61, 542-551.

Hafter, E. R., \& Carrier, S. C. Masking-level differences obtained with a pulsed tonal masker. Journal of the Acoustical Society of America, 1970, 47, 1041-1047.

Hafter, E. R., \& Carrier, S. C. Binaural interaction in lowfrequency stimuli: The inability to trade time and intensity completely. Journal of the Acoustical Society of America, 1972, 51, 1852-1862.

Jeffress, L. A., \& McFadden, D. Differences of interaural phase and level in detection and lateralization. Journal of the Acoustical Society of America, 1971, 49, 1169-1179.

McFadden, D., Jeffress, L. A., \& Ermey, H. L. Differences of interaural phase and level in detection and lateralization: $250 \mathrm{~Hz}$. Journal of the Acoustical Society of America, 1971, 50, 1484-1493.

McFadden, D., Jeffress, L. A., \& Lakey, J. R. Differences of interaural phase and level in detection and lateralization: $1000 \mathrm{~Hz}$ and $2000 \mathrm{~Hz}$. Journal of the Acoustical Society of America, 1972, 52, 1197-1206.

Osman, E. A correlation model of binaural masking level differences. Journal of the Acoustical Society of America, 1971, 50, 1494-1511.

Osman, E. Correlation model of binaural detection: Interaural amplitude ratio and phase variation for signal. Journal of the Acoustical Society of America, 1973, 54, 386-389.

Osman, E., Schacknow, P. N., \& Tzuo, P. L. Psychometric functions and a correlation model of binaural detection. Perception \& Psychophysics, 1973, 54, 386-389.

Osman, E., Tzuo, H., \& Tzuo, P. L. Theoretical analysis of detection of monaural signals as a function of interaural noise correlation and signal frequency. Journal of the Acoustical Society of America, 1975, 57, 939-942.

Osman, E., Tzuo, H., \& Tzuo, P. L. Weber's law, the "nearmiss," and binaural detection. Perception \& Psychophysics, $1980,28,354-364$.

Robinson, D. E., Langford, T. L., \& Yost, W. A. Masking of tones by tones and of noise by noise. Perception \& Psychophysics, 1974, 15, 159-167.

STERN, R. M., JR. Lateralization, discrimination and detection of binaural pure tones. Unpublished doctoral dissertation, M.I.T., 1976.

Wightman, F. L. Detection of binaural tones as a function of masker bandwidth. Journal of the Acoustical Society of America, $1971,50,623-636$.

Yost, W. A. Weber's fraction for the intensity of pure tones presented binaurally. Perception \& Psychophysics, 1972, 11, 61-64. 


\section{NOTES}

1. We chose to use $\Delta \mathrm{I}_{R} / \mathrm{I}_{R}$ (as defined in Figure 1) for the ordinate of Figure 2, as in Osman, Tzuo, and Tzuo (1980) (which also shows data of Listener H.T.) and Yost (1972). The use of $S_{R} / M$ may be conventional, but that quantity does not represent any real measure of the stimulus at the ear independently of $\alpha$. The resultant is the stimulus, and it is the energy increment of the resultant that is most important for monaural intensity discrimination. Thus, the presentation of $\Delta \mathbf{I}_{R} / \mathbf{I}_{R}$ in Figure 2 is informative, since it makes clear the inadequacy of that quantity as the basis of discrimination under the binaural conditions.
2. Note that the binaural diotic masked threshold is lower than either of the monaural masked thresholds. The monaural masked thresholds show a right-ear advantage for all four listeners. This is mentioned since it is an interaural weighting difference, which may partly determine the antiphasic masking results.

3. Given the theoretical issue and the consequent design of the experiment, there is no logically proper way to estimate psychometric functions (or their corresponding thresholds) for the second (uncertainty) stage.

(Manuscript received March 30, 1981;

revision accepted for publication January 16, 1982.) 